\title{
Observation on Curative Effect and Adverse Reaction of Acupuncture and Moxibustion in Treating Chronic Functional Constipation
}

\author{
Jiayi Lu \\ Chengdu University of TCM, Chengdu 610000, Sichuan, China \\ Email:2417953769@qq.com
}

\begin{abstract}
Objective - To investigate the efficacy of acupuncture and moxibustion in the treatment of chronic functional constipation and the observation of adverse reactions in patients. Methods - A total of 88 patients with chronic functional constipation who were treated from June 2019 to March 2021 were selected as the subjects, and the patients were divided into control group with 44 patients and observation group with 44 patients according to a random number table.The control group was given conventional western medication, and the observation group was given acupuncture based treatment. The scores for clinical symptom and the incidence of adverse reactions were compared between the two groups after treatment. Results - After treatment, the symptom scores of frequency of abdominal pain, incomplete sense of defecation, abdominal distension and difficulty in defecation in the observation group were all lower than those in the control group (P $<0.05)$. After treatment, the total incidence of adverse reactions of abdominal distension, spasmodic abdominal pain, borborygmus and dizziness in observation group was $4.55 \%$, which was significantly lower than that in control group, 22.73\% (P<0.05). Conclusion - Acupuncture and moxibustion therapy is more effective than conventional western medicine therapy in treatment of chronic functional constipation, which effectively improve the clinical symptoms of patients, reduce the occurrence of adverse reactions, as as result, it is worthy of promotion and universal application.
\end{abstract}

Keywords: acupuncture, chronic functional constipation, efficacy, adverse reactions

Compared with organic constipation, functional constipation mainly refers to abnormal humoral regulation or neuroregulation caused by poor defecation, leading to changes in intestinal and gastrointestinal functions, which are manifested as difficult defecation, reduced frequency of defecation, long duration of defecation, endless frequency of defecation, etc., seriously affecting the work and life of patients ${ }^{[1]}$. Functional constipation not only affects the patient's appetite and mental state, but also affects the patient's quality of life. With the continuous development of medical technology, acupuncture and moxibustion has been widely used in the treatment of patients with functional constipation with ideal therapeutic effects ${ }^{[2]}$, but few relevant studies were conducted. Therefore, this study conducted a study on patients with chronic functional constipation under treatment, and discussion was conducted on the efficacy of acupuncture and moxibustion in the treatment of chronic functional constipation and the observation of adverse reactions in patients, which is reported as follows.

\section{Data and methods}

\subsection{Clinical data}

A total of 88 patients with chronic functional constipation who were treated from June 2019 to March 2021 were selected as the subjects, and the patients were divided into control group with 44 patients and observation group with 44 patients using a random number table. In the control group, there were 19 males and 25 females, aged (43-76) years old, with an average of $(62.49 \pm 5.71)$ years old. The course of disease was (6d-3) years, with an average of $(1.35 \pm 0.44)$ years. The body mass index was $(20-26) \mathrm{kg} / \mathrm{m}^{2}$, with an average of $(23.10 \pm 0.71) \mathrm{kg} / \mathrm{m}^{2}$. In observation group, there were 20 males and 24 females, aged (45-75) years old, with an average of (61.81 \pm 5.51$)$ years old.The course of disease was (7d-3) years, with an average of $(1.69 \pm 0.25)$ years. The body mass index was $(21-25) \mathrm{kg} / \mathrm{m}^{2}$, with an average of $(23.46 \pm 0.78) \mathrm{kg} / \mathrm{m}^{2}$.

\subsection{Inclusion and exclusion criteria}

Inclusion criteria: (1) All were clinical diagnostic criteria for functional constipation, with clinical symptoms such as reduced frequency of defecation, labored defecation, and sense of incomplete defecation ${ }^{[3]}$; (2) The condition was stable without other cardiovascular diseases. Exclusion criteria: (1) Patients with other immune diseases, malignant tumors or incomplete medical records; (2) People with infectious diseases. 


\subsection{Methods}

Control group was given oral cisapride tablet (State Food and Drug Administration Approval Number: H20020345, manufacturer: Zhejiang Jingxin Pharmaceutical Co.,Ltd.) for treatment, the daily total of 15-30mg, 2-3 times before meals / $15 \mathrm{~min}$ before going to bed, 3 weeks as a course of treatment according to the severity of the patient's condition. The observation group was treated with acupuncture and moxibustion, and Tianshu, Zhigou, Yaodi, Erbai, Shangjuxu, Dachang Shu and Zusanli acupoints were selected. Hot secret type plus Hegu and Quchi points. Hegu and Quchi acupoints were selected in terms of Remi. Zhongwan and Qihai acupoints were selected in terms of Qimi acupoints. Shi Guan acupoints and Zhao Hai acupoints were selected for Lengmi. According to the deficiency and deficiency of patients, acupuncture and moxibustion were performed by twirling and tonifying method, reducing method and flattening and reducing method respectively. For the deficiency and cold secret type, warm acupuncture and moxibustion were used, leaving the needle for 30 minutes, once a day, 10 times as a course of treatment, and the interval of the course of treatment was 2 to 3 days. After 3 courses of treatment, the curative effect was statistically analyzed.

\subsection{Observation Indicators}

(1) Scores for clinical symptom: frequency of abdominal pain, feeling of incomplete defecation, abdominal distension and difficulty in defecation were observed in the two groups. A 4-grade scoring method was adopted, and the higher the score, the more serious the clinical symptoms were ${ }^{[4]}$. (2) Incidence of adverse reactions. The incidence of abdominal distension, spasmodic abdominal pain, borborygmus, dizziness and other adverse reactions in patients of the two groups after treatment was recorded and statistically analyzed.

\subsection{Statistical analysis}

SPSS21.0 software was used for processing. The statistical data were represented by $x^{2}$ test and $\mathrm{n}(\%)$, while the measurement data were tested by $\mathrm{t}$ test and represented by $(\bar{x} \pm s)$. The difference was statistically significant $(\mathrm{P}<0.05)$.

\section{Results}

\subsection{Comparison of scores for clinical symptom between the two groups}

There was no significant difference in clinical symptom scores between the two groups before treatment $(\mathrm{P}>0.05$. After treatment, the symptom scores of frequency of abdominal pain, endless defecation, abdominal distension and difficulty in defecation in the observation group were all lower than those in the control group $(\mathrm{P}<0.05)$, as shown in Table 1 .

\begin{tabular}{|c|c|c|c|c|c|}
\hline \multicolumn{2}{|c|}{ Groups } & \multirow{2}{*}{$\begin{array}{c}\begin{array}{c}\text { Frequency of } \\
\text { abdominal pain }\end{array} \\
2.41 \pm 0.61\end{array}$} & \multirow{2}{*}{$\begin{array}{c}\begin{array}{c}\text { Sense of incomplete } \\
\text { defecation }\end{array} \\
3.31 \pm 0.77\end{array}$} & \multirow{2}{*}{$\begin{array}{c}\text { abdominal distension } \\
3.41 \pm 0.18\end{array}$} & \multirow{2}{*}{$\begin{array}{c}\text { Difficult defecation } \\
3.42 \pm 1.03\end{array}$} \\
\hline Observation group & Pr-treatment & & & & \\
\hline$(n=44)$ & Post-treatment & $0.63 \pm 0.12^{\mathrm{ab}}$ & $0.77 \pm 0.20^{\mathrm{ab}}$ & $0.45 \pm 0.05^{\mathrm{ab}}$ & $0.23 \pm 0.04^{\mathrm{ab}}$ \\
\hline \multirow{2}{*}{$\begin{array}{l}\text { Control group } \\
\qquad(\mathrm{n}=44)\end{array}$} & Pr-treatment & $2.40 \pm 0.62$ & $3.30 \pm 0.76$ & $3.52 \pm 1.12$ & $3.43 \pm 1.04$ \\
\hline & Post-treatment & $1.36 \pm 0.34^{\mathrm{b}}$ & $1.29 \pm 0.23^{\mathrm{b}}$ & $1.23 \pm 0.34^{\mathrm{b}}$ & $1.49 \pm 0.08^{\mathrm{b}}$ \\
\hline
\end{tabular}

Compared with the control group, ${ }^{a} P<0.05$; Compared with pr-treatment, ${ }^{\mathrm{b}} P<0.05$

\subsection{Comparison of the incidence of adverse reactions between the two groups}

After treatment, the total incidence of adverse reactions of abdominal distension, spasmodic abdominal pain, enteritis and dizziness was $4.55 \%$ in the observation, which was significantly lower than that in the control group, $22.73 \%(\mathrm{P}<0.05)$, as shown in Table 2.

Table 2. Comparison of incidence of adverse reactions between the two groups [n (\%)]

\begin{tabular}{|c|c|c|c|c|c|c|}
\hline Groups & Cases & Abdominal Distension & Spasmodic Colic & Borborygmus & Dizziness & Total Incidence \\
\hline Observation group & 44 & $1(2.27)$ & $0(0.00)$ & $1(2.27)$ & $0(0.00)$ & $2(4.55)$ \\
\hline Control group & 44 & $3(6.82)$ & $2(4.55)$ & $3(6.82)$ & $2(4.55)$ & $10(22.73)$ \\
\hline$x^{2}$ & / & & & & & 6.891 \\
\hline $\mathrm{P}$ & l & & & & & 0.021 \\
\hline
\end{tabular}




\section{Discussion}

With the rapid development of society, the incidence of functional constipation is increasing year by year due to factors such as fast pace of life and irregular diet. The incidence of this disease is high among the elderly population with complex pathogenesis, and the inducing factors are mostly related to the poor living habits, dietary structure and physical factors of the elderly ${ }^{[5]}$. Functional constipation in young and middle-aged adults can be alleviated by improving their living and dietary habits, while functional constipation in the elderly is stubborn and prone to recurrent episodes, which is difficult to be alleviated by improving their living and dietary habits. Therefore, reasonable clinical treatment is required ${ }^{[6]}$.

Traditional Chinese medicine believes that if the disease is caused by the stomach and intestine, Neijie, Qi deficiency, blood deficiency or intestinal dryness and other symptoms, constipation will occur. Its pathogenic site is in the intestine with various etiology, but all is caused by the conduction dysfunction of the large intestine. Treatment should be given with adjustment of Fu Qi and relaxing bowels defecation. Tianshu acupoint and large intestine are closely related, which can Jie Yang Ming Qi and alleviate stagnation. The upper Juxu acupoint is the lower acupoint, which regulates the stomach and promotes the Sanjiao Qi mechanism. Tianshu acupoint can regulate spleen and stomach function, increase intestinal peristalsis. Chigou acpoints, large intestine acpoints can adjust the Sanjiao Qi machine, which can circulate the meridians. Yaoqi acupoint and Erbai acupoint are extra nerve points, which can which can improve constipation and hemorrhoids. The shu point and quchi point of the large intestine together with the original point can play the role of clearing the fire of the stomach and intestines, reducing heat and relieving constipation. Fu Hui Zhong Wan point with Qi sea point can pass down Fu Qi; Pishu point and Wei Yu point together, toning can regulate the spleen and stomach. Shiguan acupoint and Zhaohai acupoint together, can contact the surface, clear and reduce turbidity. Warm acupuncture and moxibustion can effectively enhance the temperature-tonic effect ${ }^{[7]}$. In this study, after treatment, the symptom scores of frequency of abdominal pain, insufficiency of defecation, abdominal distension and difficulty in defecation in the observation group were all lower than those in the control group $(\mathrm{P}<0.05)$, indicating that acupuncture and moxibustion treatment of chronic functional constipation can effectively improve the clinical symptoms of patients and promote recovery. Among the traditional western medicines, cisapride is a gastrointestinal motility drug, which can selectively promote the release of acetylcholine after the intestinal myogonia, thus enhancing gastrointestinal motility and playing a certain role in the treatment of constipation. However, it can cause different degrees of adverse reactions in patients ${ }^{[8]}$. In this study, the observation group of patients after treatment of abdominal distension, spastic abdominal pain, bowel, dizziness adverse reactions to the total incidence was $4.55 \%$, significantly lower than the control group $22.73 \%$ ( $(\mathrm{P}<0.05)$, the acupuncture treatment of chronic functional constipation higher than conventional medication safety, produce less adverse reaction, can effectively improve the patients' treatment compliance.

In conclusion, the curative effect of acupuncture on chronic functional constipation is more obvious than that of conventional western medicine, which can effectively improve the clinical symptoms of patients, reduce the occurrence of adverse reactions, as a result, it is worthy of promotion and application.

\section{References}

[1] Pan Yuezhi, Qiu Ting, Xu Yujing. Analysis of nursing operation and application effect of Keserlox in the treatment of functional constipation[J]. Hainan Medical Journal,2019,30(8):1086-1088.

[2] Zhang Xiaohui, Xue Qiming, Yong Fengiiao, et al. Clinical evaluation of acupoint catgut embedding in the treatment of functional constipation[J]. Sichuan Traditional Chinese Medicine,2019,37;433 (12) : 223-225.

[3] Bu Fan, Li Mengyi, Gu Yunfei. Clinical observation of Jichuan decoction combined with Zhizhu pill in the treatment of middle-aged and elderly chronic functional constipation[J]. Modern Journal of Integrated Traditional Chinese and Western Medicine,2019,28(1):23-26.

[4] Zhang Jing, Gu Zhiju. Effect comparison of different intestinal preparation methods in enteroscopy of chronic functional constipation in the elderly[J]. Modern Digestion \& Intervention,2019,24(11):101-105.

[5] Kuang Xiaowen, Zheng Yuxiang, Gong Yuju. Clinical analysis of Jichuan decoction in elderly patients with chronic functional constipation[J]. Journal of Colorectal \& Anal Surgery,2019,25(5):100-105.

[6] Tan Yi, Yuan Longjian, Fu Zhonghua. Clinical efficacy of floating needle therapy in the treatment of functional constipation[J]. China Medical Herald,2019,16(27):155-158.

[7] Liu Haiquan, Tan Rui. Treatment of functional constipation in 48 elderly patients with yiqi qing-heat decoction combined with sarcopyridine[J]. Chinese Journal of Multiple Organ Diseases in the Elderly,2020,19(6):62-63.

[8] Bu Fan, Li Mengyi, Gu Yunfei. Clinical observation of Jichuan decoction combined with Zhizhu pill in the treatment of middle-aged and elderly chronic functional constipation[J]. Modern Journal of Integrated Traditional Chinese and Western Medicine,2019,28(1):23-26. 